\title{
An efficient route towards the synthesis of monosubstituted $N$-aryl amidines from 4,5-dihydro-1,2,4-oxadiazoles
}

\author{
Neelam L. Chavan ${ }^{a}$, Nilesh H. Naik ${ }^{a}$, Sandip K. Nayak ${ }^{b}$ and Radhika S. Kusurkar ${ }^{a, *}$ \\ ${ }^{a}$ Department of Chemistry, University of Pune, Pune, 411 007, India \\ ${ }^{b}$ Bio-organic Division, Bhabha Atomic Research Centre, Mumbai, 400 085, India \\ E-mail: rsk@chem.unipune.ernet.in
}

\begin{abstract}
4,5-Dihydro-1,2,4-oxadiazoles were prepared using 1,3-dipolar cycloaddition of imines with nitrile oxides. Further reductive N-O bond cleavage furnished monosubstituted $N$-aryl amidines in good yield. Thus an efficient route for the synthesis of monosubstituted $N$-aryl amidines has been developed.
\end{abstract}

Keywords: $N$-aryl amidines, 4,5-dihydro-1,2,4-oxadiazoles, hydrogenolysis, Nitrile oxides, 1,3dipolar cycloaddition reaction

\section{Introduction}

$N$-Aryl amidines are important synthons for the synthesis of several heterocyclic compounds. ${ }^{1}$ These compounds show a wide spectrum of biological activity like antibacterial, antimicrobial, anticancer and antiviral activity. ${ }^{2}$ As substituted amidines have significance in several biologically active compounds, their synthesis has been of interest for several research groups. ${ }^{3}$ Amongst the reported synthetic strategies, nucleophilic addition of amine to nitrile is one of the important methods. Various Lewis acids like trivalent lanthanide triflates, ${ }^{3 \mathrm{e}} \mathrm{SmI}_{2},{ }^{3 \mathrm{f}} \mathrm{CuCl},{ }^{3 \mathrm{~g}}$ etc. have been used as catalysts. The development of catalytic addition of amines to nitriles is still a challenge in monosubstituted amidine synthesis. Some other methods for the synthesis of monosubstituted amidines are reaction of nitrile and azide catalyzed by $\mathrm{Sm}^{3 \mathrm{~h}}$ and reaction of nitrile and azo compound catalyzed by $\mathrm{SmI}_{2}{ }^{31}$ Considering the need of a good route towards the synthesis of monosubstituted amidines, it was planned to use the N-O bond cleavage reaction of 4,5-dihydro-1,2,4-oxadiazoles for this purpose. 


\section{Results and Discussion}

1,3-Dipolar cycloaddition reactions have been used in our laboratory for the synthesis of various isoxazolidines ${ }^{4}$ and 2 -isoxazolines. ${ }^{5}$ Similarly, preparation of 5-aryl-4,5-dihydro-1,2,4oxadiazoles via 1,3-dipolar cycloaddition of nitrile oxides and imines has already been reported. ${ }^{6}$ Bolton et $\mathrm{al}^{7}$ reported a route to mono-substituted amidines via cleavage of $\mathrm{N}-\mathrm{O}$ bond of 1,2,4oxadiazolin-5-ones under catalytic hydrogenation conditions.

Keeping above reported reactions in view, we envisaged that the $\mathrm{N}-\mathrm{O}$ bond cleavage in 5aryl-4,5-dihydro-1,2,4-oxadiazoles might lead to the formation of either $\mathrm{N}$ (hydroxy(aryl)methyl)- $N$-arylbenzamides 27 produced by hydrolysis of a first-formed imine or $\mathrm{N}$-aryl amidines (Scheme 1). We herein report the formation of monosubstituted $\mathrm{N}$-aryl amidines during the reductive $\mathrm{N}-\mathrm{O}$ bond cleavage of 4,5-dihydro-1,2,4-oxadiazoles.

The aromatic imines 5-9 were prepared following a reported procedure. ${ }^{8} 4,5$-Dihydro-1,2,4oxadiazoles were synthesized using 1,3-dipolar cycloaddition reaction. Thus benzaldehyde oxime 1 was converted into the corresponding benzohydroximoyl chloride with NCS. Treatment of the mixture of benzohydroximoyl chloride and imine 5 with triethylamine at $0{ }^{\circ} \mathrm{C}$ afforded the expected 4,5-dihydro-1,2,4-oxadiazole $\mathbf{1 0}$ in 65\% yield. Similarly, other 4,5-dihydro-1,2,4oxadiazoles 11-19 were prepared in good yields (Table 1).

In a model study on the hydrogenolysis, oxadiazole 10 with Raney nickel in methanol at room temperature was exposed to hydrogen gas (balloon pressure) to afford a single product. The product was purified by column chromatography and its structure was assigned as substituted amidine 20 from its NMR spectral data and by analogy with the hydrogenation reaction reported earlier. ${ }^{7}$ The probable mechanism of formation of $\mathbf{2 0}$ can be explained by the reductive cleavage of $\mathrm{N}-\mathrm{O}$ bond to $\mathrm{N}$-(hydroxy(aryl)methyl)benzamidine with hydrogenolysis of benzylic alcohol to furnish amidine $\mathbf{2 0}$ (Scheme 2). 


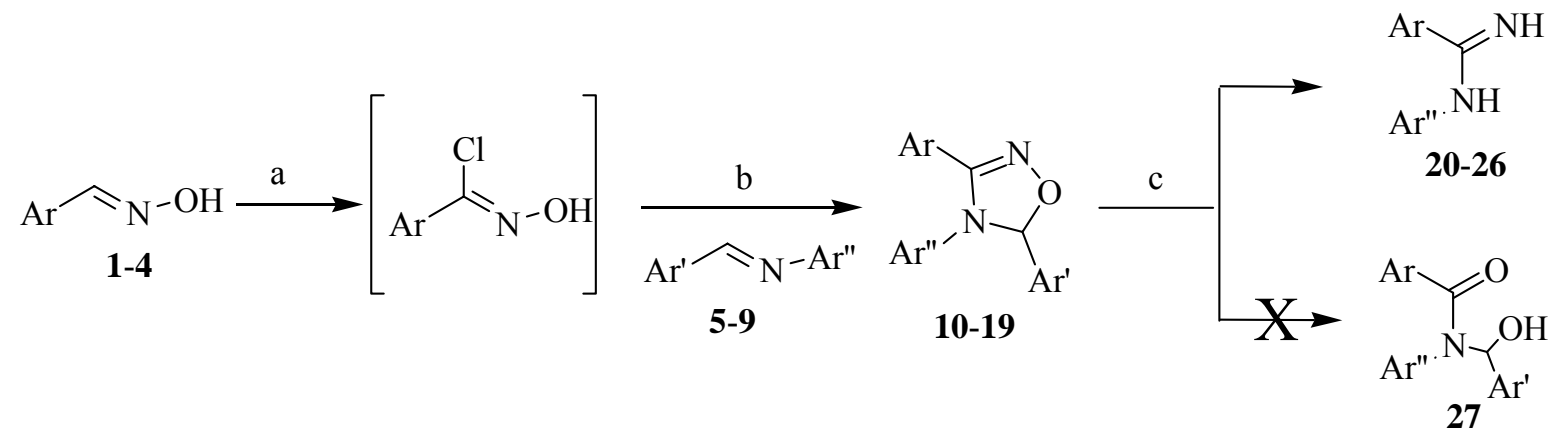

a. NCS, DCM, r.t., 2 h ; b. $\mathrm{NEt}_{3}$, DCM, $0^{\circ} \mathrm{C}-$ r.t., 1 h ; c. Raney nickel, $\mathrm{H}_{2}$, MeOH, r.t., balloon pressure, $2-3 \mathrm{~h}$

\begin{tabular}{|c|c|c|c|}
\hline Compound No. & Ar & Ar' & Ar' \\
\hline 1 & $\mathrm{Ph}$ & - & - \\
\hline 2 & $4-\mathrm{MeOC}_{6} \mathrm{H}_{4}$ & - & - \\
\hline 3 & $4-\mathrm{ClC}_{6} \mathrm{H}_{4}$ & - & - \\
\hline 4 & 3,4- $\mathrm{MeOC}_{6} \mathrm{H}_{4}$ & - & - \\
\hline 5 & $\mathrm{H}$ & $\mathrm{Ph}$ & $\mathrm{Ph}$ \\
\hline 6 & $\mathrm{H}$ & $4-\mathrm{MeOC}_{6} \mathrm{H}_{4}$ & $\mathrm{Ph}$ \\
\hline 7 & $\mathrm{H}$ & $3,4-\mathrm{MeOC}_{6} \mathrm{H}_{4}$ & $\mathrm{Ph}$ \\
\hline 8 & $\mathrm{H}$ & $4-\mathrm{NO}_{2} \mathrm{C}_{6} \mathrm{H}_{4}$ & $\mathrm{Ph}$ \\
\hline 9 & $\mathrm{H}$ & $4-\mathrm{NO}_{2} \mathrm{C}_{6} \mathrm{H}_{4}$ & $4-\mathrm{MeOC}_{6} \mathrm{H}_{4}$ \\
\hline 10 & $\mathrm{Ph}$ & $\mathrm{Ph}$ & $\mathrm{Ph}$ \\
\hline 11 & $\mathrm{Ph}$ & $3,4-\mathrm{MeOC}_{6} \mathrm{H}_{4}$ & $\mathrm{Ph}$ \\
\hline 12 & $\mathrm{Ph}$ & $4-\mathrm{NO}_{2} \mathrm{C}_{6} \mathrm{H}_{4}$ & $\mathrm{Ph}$ \\
\hline 13 & $4-\mathrm{MeOC}_{6} \mathrm{H}_{4}$ & $4-\mathrm{MeOC}_{6} \mathrm{H}_{4}$ & $\mathrm{Ph}$ \\
\hline 14 & $4-\mathrm{MeOC}_{6} \mathrm{H}_{4}$ & $4-\mathrm{NO}_{2} \mathrm{C}_{6} \mathrm{H}_{4}$ & $\mathrm{Ph}$ \\
\hline 15 & $4-\mathrm{ClC}_{6} \mathrm{H}_{4}$ & $4-\mathrm{NO}_{2} \mathrm{C}_{6} \mathrm{H}_{4}$ & $\mathrm{Ph}$ \\
\hline 16 & $3,4-\mathrm{MeOC}_{6} \mathrm{H}_{4}$ & $4-\mathrm{NO}_{2} \mathrm{C}_{6} \mathrm{H}_{4}$ & $\mathrm{Ph}$ \\
\hline 17 & $3,4-\mathrm{MeOC}_{6} \mathrm{H}_{4}$ & $4-\mathrm{NO}_{2} \mathrm{C}_{6} \mathrm{H}_{4}$ & 4- $\mathrm{MeOC}_{6} \mathrm{H}_{4}$ \\
\hline 18 & $4-\mathrm{MeOC}_{6} \mathrm{H}_{4}$ & $4-\mathrm{NO}_{2} \mathrm{C}_{6} \mathrm{H}_{4}$ & $4-\mathrm{MeOC}_{6} \mathrm{H}_{4}$ \\
\hline 19 & $4-\mathrm{ClC}_{6} \mathrm{H}_{4}$ & $4-\mathrm{NO}_{2} \mathrm{C}_{6} \mathrm{H}_{4}$ & $4-\mathrm{MeOC}_{6} \mathrm{H}_{4}$ \\
\hline 20 & $\mathrm{Ph}$ & - & $\mathrm{Ph}$ \\
\hline 21 & 4- $\mathrm{MeOC}_{6} \mathrm{H}_{4}$ & - & $\mathrm{Ph}$ \\
\hline 22 & $3,4-\mathrm{MeOC}_{6} \mathrm{H}_{4}$ & - & $\mathrm{Ph}$ \\
\hline 23 & 4- $\mathrm{ClC}_{6} \mathrm{H}_{4}$ & - & $4-\mathrm{MeOC}_{6} \mathrm{H}_{4}$ \\
\hline 24 & $4-\mathrm{MeOC}_{6} \mathrm{H}_{4}$ & - & $4-\mathrm{MeOC}_{6} \mathrm{H}_{4}$ \\
\hline 25 & $3,4-\mathrm{MeOC}_{6} \mathrm{H}_{4}$ & - & $4-\mathrm{MeOC}_{6} \mathrm{H}_{4}$ \\
\hline 26 & 4- $\mathrm{ClC}_{6} \mathrm{H}_{4}$ & - & $\mathrm{Ph}$ \\
\hline
\end{tabular}

Scheme 1 
Table 1. Synthesis of 1,2,4-oxadiazoles from oxime and imine via 1,3-dipolar cycloaddition

\begin{tabular}{ccccc}
\hline Oxime & Imine & Oxadiazole & $\mathrm{Mp}\left({ }^{\circ} \mathrm{C}\right)$ of Oxadiazole & Yield $(\%)$ \\
\hline $\mathbf{1}$ & $\mathbf{5}$ & $\mathbf{1 0}$ & ${\text { 76-77 }\left(\text { lit. }^{9} \text { 75-76) }\right.}$ & 65 \\
$\mathbf{1}$ & $\mathbf{7}$ & $\mathbf{1 1}$ & $64-65$ (lit. $^{10}$ 65) & 69 \\
$\mathbf{1}$ & $\mathbf{8}$ & $\mathbf{1 2}$ & $149-151\left(\right.$ lit. $\left.^{11} 148-150\right)$ & 75 \\
$\mathbf{2}$ & $\mathbf{6}$ & $\mathbf{1 3}$ & $115-117$ & 58 \\
$\mathbf{2}$ & $\mathbf{8}$ & $\mathbf{1 4}$ & $132-134$ & 63 \\
$\mathbf{3}$ & $\mathbf{8}$ & $\mathbf{1 5}$ & $114-115$ & 65 \\
$\mathbf{4}$ & $\mathbf{8}$ & $\mathbf{1 6}$ & $150-152$ & 59 \\
$\mathbf{4}$ & $\mathbf{9}$ & $\mathbf{1 7}$ & $177-178$ & 65 \\
$\mathbf{1}$ & $\mathbf{9}$ & $\mathbf{1 8}$ & Oily liquid & 59 \\
$\mathbf{3}$ & $\mathbf{9}$ & $\mathbf{1 9}$ & $130-131$ & 66 \\
\hline
\end{tabular}

To see the scope and generality of the above reaction, several other 4,5-dihydro-1,2,4oxadiazoles 11-19 were subjected to hydrogenolysis reaction. In all the cases the expected amidines 21-26 were obtained in 74-91\% yields (Table 2). Thus, it constitutes a practical and simple method for the synthesis of substituted amidines.

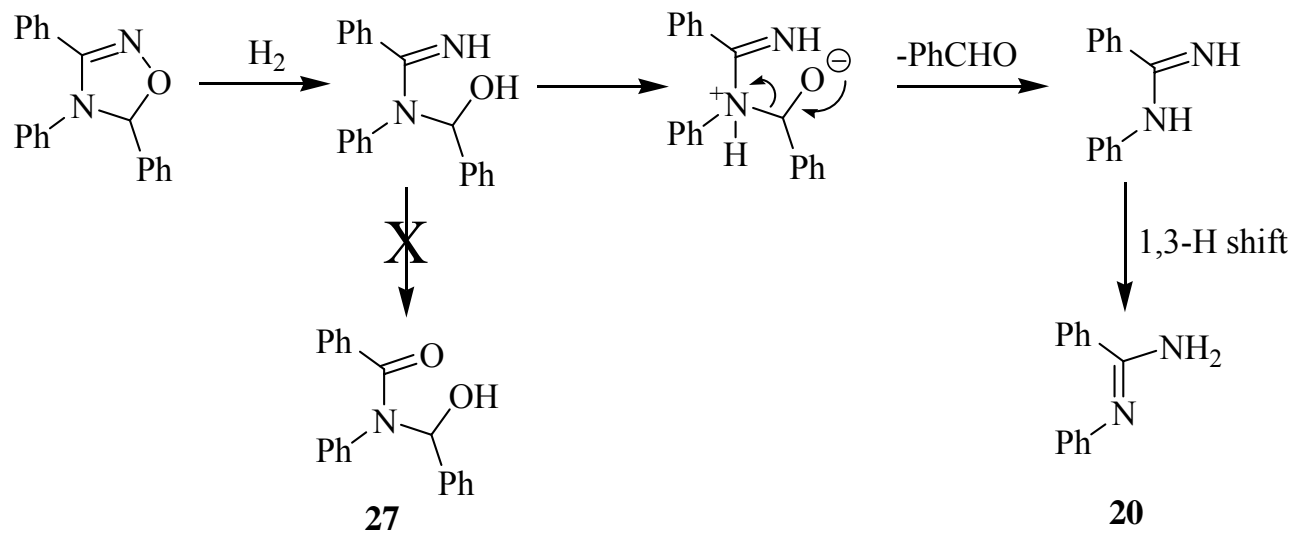

\section{Scheme 2}

Table 2. Hydrogenolysis of oxadiazoles to amidines using Raney nickel/hydrogen

\begin{tabular}{ccccc}
\hline Entry & Oxadiazole & Amidine & $\mathrm{Mp}\left({ }^{\circ} \mathrm{C}\right)$ of Amidine & Yield (\%) \\
\hline 1 & $\mathbf{1 0}$ & $\mathbf{2 0}$ & $116-118\left(\right.$ lit. $\left.^{12} 115-117\right)$ & 89 \\
2 & $\mathbf{1 1}$ & $\mathbf{2 1}$ & $137-138\left(\right.$ lit. $\left.^{13} 138-139\right)$ & 91 \\
3 & $\mathbf{1 2}$ & $\mathbf{2 2}$ & $137-138\left(\right.$ lit. $\left.^{14} 138-140\right)$ & 85 \\
4 & $\mathbf{1 3}$ & $\mathbf{2 3}$ & $138-139$ & 88 \\
\hline
\end{tabular}


Table 2. Continued

\begin{tabular}{ccccc}
\hline Entry & Oxadiazole & Amidine & Mp (oC) of Amidine & Yield (\%) \\
\hline 5 & $\mathbf{1 4}$ & $\mathbf{2 4}$ & $148-149\left(\right.$ lit. $\left.^{13} 150\right)$ & 82 \\
6 & $\mathbf{1 5}$ & $\mathbf{2 5}$ & Oily liquid & 74 \\
7 & $\mathbf{1 6}$ & $\mathbf{2 6}$ & $130-131$ & 83 \\
\hline
\end{tabular}

\section{Conclusions}

Thus, a new route for the synthesis of monosubstituted amidines, which are otherwise difficult to prepare, has been developed via hydrogenolysis of 4,5-dihydro-1,2,4-oxadiazoles. The present route is straight forward and does not yield any side product. Therefore it is a useful alternative to the existing methodologies for the synthesis of $\mathrm{N}$-aryl amidines.

\section{Experimental Section}

General. Melting points determined are uncorrected. All solvents were of reagent grade and, when necessary, were purified and dried by standard methods. Reactions and products were routinely monitored by thin layer chromatography (TLC) on silica gel (Kieselgel 60 F254, Merck). Column chromatographic purifications were performed using 100-200 mesh silica gel. IR spectra were recorded on Shimadzu 8400 instrument. ${ }^{1} \mathrm{H}$ NMR $(300 \mathrm{MHz})$ and ${ }^{13} \mathrm{C}$ NMR $(75$ $\mathrm{MHz}$ ) spectra were recorded on Varian Mercury instrument using TMS as internal standard. ${ }^{1} \mathrm{H}$ NMR peaks expressed as s, bs, d, t, m correspond to singlet, broad-singlet, doublet, triplet, and multiplet, respectively. Mass spectra were recorded on Shimadzu QP 5050. Elemental analysis was recorded on Flash E. A. 1112 Thermo instrument.

\section{General procedure for the synthesis of 4,5-dihydro-1,2,4-oxadiazoles}

In a $100 \mathrm{~mL}$ round bottom flask equipped with a guard tube, a solution of benzohydroximoyl chloride $(1 \mathrm{mmol})$ in DCM (3 mL) was placed. To this, a solution of triethylamine $(1.2 \mathrm{mmol})$ was added dropwise with vigorous stirring. During the addition, the reaction mixture became thick. To this thick reaction mixture, the imine ( $1 \mathrm{mmol}$ ) was added. Stirring was continued for $1 \mathrm{~h}$ and reaction was monitored by TLC. When the starting material was entirely consumed, water (3 $\mathrm{mL}$ ) was added to the reaction. The reaction mixture was extracted with DCM. The organic layer was washed with water and dried over sodium sulphate. The crude mixture obtained was purified by column chromatography on silica gel, where hexane-ethyl acetate (9:1) was used as an eluent. Compound 13. Yield 58\%; mp $115-117^{\circ} \mathrm{C}$; IR(KBr): $v$ 1604, $1231 \mathrm{~cm}^{-1}$; ${ }^{1} \mathrm{H}$ NMR (300 MHz, $\left.\mathrm{CDCl}_{3}\right): \delta 3.80\left(\mathrm{~s}, 3 \mathrm{H}, \mathrm{OC}_{3}\right), 3.83\left(\mathrm{~s}, 3 \mathrm{H}, \mathrm{OC}_{3}\right), 6.47(\mathrm{~s}, 1 \mathrm{H}, \mathrm{NC} \underline{\mathrm{H}}), 6.81(\mathrm{~m}, 4 \mathrm{H}, \mathrm{ArH}), 6.95$ $(\mathrm{d}, J=8.4 \mathrm{~Hz}, 2 \mathrm{H}, \mathrm{Ar} \underline{\mathrm{H}}), 7.08-7.19(\mathrm{~m}, 3 \mathrm{H}, \operatorname{Ar} \underline{\mathrm{H}}), 7.51(\mathrm{~m}, 4 \mathrm{H}, \operatorname{Ar} \underline{\mathrm{H}}) ;{ }^{13} \mathrm{C} \mathrm{NMR}(75 \mathrm{MHz}$, 
$\left.\mathrm{CDCl}_{3}\right): \delta 55.29,99.86,114.02,114.09,117.55,124.17,125.35,128.61,129.07,129.54,131.13$, 141.19, 149.85, 154.80, 160.63, 161.19; m/z: $360\left(\mathrm{M}^{+}\right)$; Anal. Calcd for $\mathrm{C}_{22} \mathrm{H}_{20} \mathrm{~N}_{2} \mathrm{O}_{3}$ : C, 73.32; H, 5.59; N, 7.77. Found: C, 73.27; H, 5.52; N, 7.73.

Compound 14. Yield $63 \%$; mp 132-134 ${ }^{\circ} \mathrm{C}$; IR(KBr): v 1602, 1517, $1218,1346 \mathrm{~cm}^{-1}$; ${ }^{1} \mathrm{H}$ NMR $\left(300 \mathrm{MHz}, \mathrm{CDCl}_{3}\right): \delta 3.80\left(\mathrm{~s}, 3 \mathrm{H}, \mathrm{OC}_{3}\right), 6.57(\mathrm{~s}, 1 \mathrm{H}, \mathrm{NC} \underline{\mathrm{H}}), 6.84(\mathrm{~m}, 3 \mathrm{H}, \mathrm{Ar} \underline{\mathrm{H}}), 7.14-7.25(\mathrm{~m}$, 4H, Ar $\underline{\mathrm{H}}$ ), 7.54 (d, $J=7.3 \mathrm{~Hz}, 2 \mathrm{H}, \operatorname{Ar} \underline{\mathrm{H}}), 7.79$ (d, $J=7.3 \mathrm{~Hz}, 2 \mathrm{H}, \operatorname{Ar} \underline{\mathrm{H}}), 8.30$ (d, J=7.3 Hz, 2H, $\operatorname{Ar} \underline{H}) ;{ }^{13} \mathrm{C}$ NMR $\left(75 \mathrm{MHz}, \mathrm{CDCl}_{3}\right): \delta 55.28,98.48,114.47,116.67,124.03,124.21,124.67$, 126.07, 127.96, 128.37, 129.44, 129.61, 141.18, 145.84, 148.62, 155.01, 161.51; m/z: $375\left(\mathrm{M}^{+}\right)$; Anal. Calcd for $\mathrm{C}_{21} \mathrm{H}_{17} \mathrm{~N}_{3} \mathrm{O}_{4}$ : C, 65.68; H, 6.61; N, 10.21. Found: C, 65.73; H, 6.65; N, 10.19 .

Compound 15. Yield $65 \%$; mp 114-115 ${ }^{\circ} \mathrm{C}$; IR(KBr): v 1621, 1562, $1349 \mathrm{~cm}^{-1} ;{ }^{1} \mathrm{H}$ NMR (300 $\left.\mathrm{MHz}, \mathrm{CDCl}_{3}\right): \delta 6.55(\mathrm{~s}, 1 \mathrm{H}, \mathrm{NC} \underline{\mathrm{H}}), 6.78(\mathrm{~d}, J=8 \mathrm{~Hz}, 2 \mathrm{H}, \mathrm{Ar} \underline{\mathrm{H}}), 7.11-7.27$ (m, 5H, Ar$\left.\underline{\mathrm{H}}\right), 7.49$ (d, $J=8 \mathrm{~Hz}, 2 \mathrm{H}, \operatorname{Ar} \underline{\mathrm{H}}), 7.73$ (d, $J=8 \mathrm{~Hz}, 2 \mathrm{H}, \operatorname{Ar} \underline{\mathrm{H}}), 8.25(\mathrm{~d}, J=8 \mathrm{~Hz}, 2 \mathrm{H}, \operatorname{Ar} \underline{\mathrm{H}}) ;{ }^{13} \mathrm{C}$ NMR $(75$ $\left.\mathrm{MHz}, \mathrm{CDCl}_{3}\right): \delta 98.95,123.05,124.00,124.38,126.48,127.96,129.01,129.57,130.38,136.84$, 140.55, 145.29, 148.62, 154.44; m/z: $379\left(\mathrm{M}^{+}\right), 381(\mathrm{M}+2)^{+}$in 3:1 ratio; Anal. Calcd for $\mathrm{C}_{20} \mathrm{H}_{14} \mathrm{ClN}_{3} \mathrm{O}_{3}$ : C, 63.25; H, 3.72; N, 11.06. Found: C, 63.19; H, 3.77; N, 11.01 .

Compound 16. Yield $59 \%$; mp 150-152 ${ }^{\circ} \mathrm{C}$; IR(KBr): v 1624, 1559, 1255, $1362 \mathrm{~cm}^{-1}$; ${ }^{1} \mathrm{H}$ NMR $\left(300 \mathrm{MHz}, \mathrm{CDCl}_{3}\right): \delta 3.78\left(\mathrm{~s}, 3 \mathrm{H}, \mathrm{OC}_{3}\right), 3.86\left(\mathrm{~s}, 3 \mathrm{H}, \mathrm{OC}_{3}\right), 6.88(\mathrm{~s}, 1 \mathrm{H}, \mathrm{NC} \underline{\mathrm{H}}), 6.58-6.88(\mathrm{~m}$, $3 \mathrm{H}, \mathrm{Ar} \underline{\mathrm{H}}), 7.07-7.28$ (m, 5H, Ar$\underline{\mathrm{H}}), 7.78-7.81$ (m, $2 \mathrm{H}, \mathrm{Ar} \underline{\mathrm{H}}), 8.28-8.31$ (m, $2 \mathrm{H}, \mathrm{Ar} \underline{\mathrm{H}}) ;{ }^{13} \mathrm{C} \mathrm{NMR}$ $\left(75 \mathrm{MHz}, \mathrm{CDCl}_{3}\right): \delta 55.76,55.91,98.60,110.40,110.70,116.68,121.46,123.96,124.42$, 126.19, 127.59, 127.94, 129.04, 141.17, 145.67, 148.81, 151.02, 155.11; m/z: $405\left(\mathrm{M}^{+}\right)$; Anal. Calcd for $\mathrm{C}_{22} \mathrm{H}_{19} \mathrm{~N}_{3} \mathrm{O}_{5}$ : C, 65.18; H, 4.72; N, 10.37. Found: C, 65.25; H, 4.67; N, 10.33 .

Compound 17. Yield $65 \%$; $\mathrm{mp} \mathrm{177-178}{ }^{\circ} \mathrm{C}$; IR(KBr): v 1628, 1539, 1267, $1336 \mathrm{~cm}^{-1}$; ${ }^{1} \mathrm{H}$ NMR (300 MHz, $\left.\mathrm{CDCl}_{3}\right): \delta 3.67\left(\mathrm{~s}, 3 \mathrm{H}, \mathrm{OC}_{3}\right), 3.72\left(\mathrm{~s}, 3 \mathrm{H}, \mathrm{OC}_{3}\right), 3.79\left(\mathrm{~s}, 3 \mathrm{H}, \mathrm{OC}_{3}\right), 6.43(\mathrm{~s}, 1 \mathrm{H}$

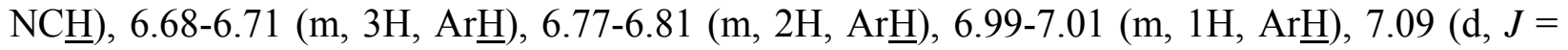
$1.9 \mathrm{~Hz}, 1 \mathrm{H}, \operatorname{Ar} \underline{\mathrm{H}}), 7.71$ (d, $J=6.8 \mathrm{~Hz}, 2 \mathrm{H}, \operatorname{Ar} \underline{\mathrm{H}}), 8.20-8.23(\mathrm{~m}, 2 \mathrm{H}, \operatorname{Ar} \underline{\mathrm{H}}) ;{ }^{13} \mathrm{C} \mathrm{NMR}(75 \mathrm{MHz}$, $\left.\mathrm{CDCl}_{3}\right): \delta 55.60,56.05,99.57,110.84,110.95,114.99,117.02,121.75,124.19,127.46,128.48$, 133.95, 145.98, 149.00, 151.15, 156.03, 158.49; m/z: $435\left(\mathrm{M}^{+}\right)$; Anal. Calcd for $\mathrm{C}_{23} \mathrm{H}_{21} \mathrm{~N}_{3} \mathrm{O}_{6}: \mathrm{C}$, 63.44; H, 4.86; N, 9.65. Found: C, 63.38; H, 4.81; N, 9.58.

Compound 18. Yield $59 \%$; Oily liquid; IR(KBr): $v 1625,1534,1245,1323 \mathrm{~cm}^{-1} ; \mathrm{H}$ NMR (300 $\left.\mathrm{MHz}, \mathrm{CDCl}_{3}\right): \delta 3.74\left(\mathrm{~s}, 3 \mathrm{H}, \mathrm{OC}_{\mathrm{H}_{3}}\right), 3.77\left(\mathrm{~s}, 3 \mathrm{H}, \mathrm{OC}_{3}\right), 6.44(\mathrm{~s}, 1 \mathrm{H}, \mathrm{NC} \underline{\mathrm{H}}), 6.75-6.81(\mathrm{~m}, 4 \mathrm{H}$, $\mathrm{ArH}), 6.91(\mathrm{~d}, J=8.8 \mathrm{~Hz}, 2 \mathrm{H}, \operatorname{Ar} \underline{\mathrm{H}}), 7.02-7.14(\mathrm{~m}, 2 \mathrm{H}, \mathrm{Ar} \underline{\mathrm{H}}), 7.46-7.50$ (m, $4 \mathrm{H}, \operatorname{Ar} \underline{\mathrm{H}}) ;{ }^{13} \mathrm{C} \mathrm{NMR}$ $\left(75 \mathrm{MHz}, \mathrm{CDCl}_{3}\right): \delta 55.57,100.10,114.32,117.74,124.39,125.57,128.51,128.80,129.27$, 129.71, 131.26, 141.33, 154.93, 160.76, 161.33; m/z: $405\left(\mathrm{M}^{+}\right)$; Anal. Calcd for $\mathrm{C}_{15} \mathrm{H}_{18} \mathrm{~N}_{2} \mathrm{O}_{3}$ requires: $\mathrm{C}, 65.18 ; \mathrm{H}, 4.72 ; \mathrm{N}, 10.37$. Found: $\mathrm{C}, 65.23 ; \mathrm{H}, 4.69 ; \mathrm{N}, 10.34$.

Compound 19. Yield $66 \%$; mp 130-131 ${ }^{\circ} \mathrm{C}$; IR(KBr): $v$ 1610, 1597, 1243, $1344 \mathrm{~cm}^{-1} ;{ }^{1} \mathrm{H}$ NMR (300 MHz, $\left.\mathrm{CDCl}_{3}\right): \delta 3.74$ (s, 3H, $\left.\mathrm{OC}_{3}\right), 6.48(\mathrm{~s}, 1 \mathrm{H}, \mathrm{NC} \underline{\mathrm{H}}), 6.73-6.82(\mathrm{~m}, 4 \mathrm{H}, \mathrm{ArH}), 7.28$ (d, J $=8.2 \mathrm{~Hz}, 2 \mathrm{H}, \operatorname{Ar} \underline{\mathrm{H}}), 7.49$ (d, $J=8.2 \mathrm{~Hz}, 2 \mathrm{H}, \operatorname{Ar} \underline{\mathrm{H}}), 7.75(\mathrm{~d}, J=8.7 \mathrm{~Hz}, 2 \mathrm{H}, \operatorname{Ar} \underline{\mathrm{H}}), 8.29$ (d, $J=$ $8.7 \mathrm{~Hz}, 2 \mathrm{H}, \mathrm{Ar} \underline{\mathrm{H}}) ;{ }^{13} \mathrm{C} \mathrm{NMR}\left(75 \mathrm{MHz}, \mathrm{CDCl}_{3}\right): \delta 55.36,99.71,114.90,123.11,124.00,127.19$, $128.22,128.95,129.26,133.03,136.75,145.33,155.19,158.44 ; \mathrm{m} / \mathrm{z}: 409\left(\mathrm{M}^{+}\right), 411(\mathrm{M}+2)^{+}$in 
3:1 ratio; Anal. Calcd for $\mathrm{C}_{21} \mathrm{H}_{16} \mathrm{ClN}_{3} \mathrm{O}_{4}$ : C, 61.54; H, 3.94; N, 10.25. Found: C, $61.52 \mathrm{H}, 3.89$; $\mathrm{N}, 10.22$.

\section{General procedure for the synthesis of $\mathrm{N}$-aryl amidines}

To a solution of the 4,5-dihydro-1,2,4-oxadiazole (1 mmol) in methanol $(5 \mathrm{~mL})$, Raney nickel (10 mol\%) was added and the mixture was stirred (2-3 h) under hydrogen atmosphere using balloon at room temperature. The progress of the reaction was followed by TLC. After completion of reaction, the mixture was filtered through celite bed and the filtrate was concentrated on a rotavapor. The crude product was purified by column chromatography using hexane-ethyl acetate (1:1) as an eluent and recrystallized with methanol-water solvent system.

Compound 23. Yield $88 \%$; mp 138-139 ${ }^{\circ} \mathrm{C}$; IR (KBr): $v$ 3333, 1613, $1243 \mathrm{~cm}^{-1}$; ${ }^{1} \mathrm{H}$ NMR $\left(\mathrm{CDCl}_{3}\right): \delta 3.77\left(\mathrm{~s}, 3 \mathrm{H}, \mathrm{OC}_{3}\right), 5.04$ (bs, $\left.2 \mathrm{H}, \mathrm{N}_{2}\right), 6.85$ (bs, $\left.4 \mathrm{H}, \mathrm{ArH}\right), 7.33$ (d, $2 \mathrm{H}, \mathrm{J}=8.2 \mathrm{~Hz}$, $\operatorname{Ar} \underline{\mathrm{H}}), 7.71$ (d, $2 \mathrm{H}, \mathrm{J}=8.2 \mathrm{~Hz}, \operatorname{Ar} \underline{\mathrm{H}}) ;{ }^{13} \mathrm{C} \mathrm{NMR}\left(75 \mathrm{MHz}, \mathrm{CDCl}_{3}\right.$, Fig. No. 10b): $\delta 55.33,114.74$, 122.47, 128.14, 128.48, 133.99, 136.37, 141.64, 154.65, 155.62; $\mathrm{m} / \mathrm{z} 260\left(\mathrm{M}^{+}\right), 262\left(\mathrm{M}^{+}+2\right)^{+}$in 3:1 ratio; Anal. Calcd for $\mathrm{C}_{14} \mathrm{H}_{13} \mathrm{ClN}_{2} \mathrm{O}: \mathrm{C}, 64.49 ; \mathrm{H}, 5.03 ; \mathrm{N}, 10.74$. Found: $\mathrm{C}, 64.53 ; \mathrm{H}, 5.07$; $\mathrm{N}, 10.79$.

Compound 25. Yield $74 \%$; Oily liquid; IR (neat): $v$ 3387, $1614 \mathrm{~cm}^{-1} ;{ }^{1} \mathrm{H}$ NMR $(300 \mathrm{MHz}$, $\mathrm{CDCl}_{3}$ ): $\delta 3.16$ (bs, $2 \mathrm{H}, \mathrm{N}_{2}$ ), 4.02 (s, 9H, 3xOC$\left.\underline{H}_{3}\right), 6.67-6.72$ (m, 3H, $\left.\mathrm{Ar} \underline{\mathrm{H}}\right), 7.04$ (d, $1 \mathrm{H}, J=$ $4.5 \mathrm{~Hz}, \operatorname{Ar} \underline{\mathrm{H}}), 7.18-7.29$ (m, 3H, $\mathrm{Ar} \underline{\mathrm{H}}) ;{ }^{13} \mathrm{C} \mathrm{NMR}\left(75 \mathrm{MHz}, \mathrm{CDCl}_{3}\right): \delta 55.29,114.23,114.83$, 122.64, 128.23, 128.44, 128.76, 155.41, 161.32; $\mathrm{m} / \mathrm{z} 286\left(\mathrm{M}^{+}\right)$; Anal. Calcd for $\mathrm{C}_{16} \mathrm{H}_{18} \mathrm{~N}_{2} \mathrm{O}_{3}: \mathrm{C}$, 67.12; H, 6.34; N, 9.78. Found: C, 67.07; H, 6.29; N, 9.83.

Compound 26. Yield $83 \%$; mp 130-131 ${ }^{\circ} \mathrm{C}$; IR (neat): $v 3308,1616 \mathrm{~cm}^{-1} ;{ }^{1} \mathrm{H}$ NMR $(300 \mathrm{MHz}$, $\mathrm{CDCl}_{3}$ ): $\delta 5.78$ (bs, $2 \mathrm{H}, \mathrm{N}_{2}$ ), 6.92-7.01 (m, 3H, $\left.\mathrm{Ar} \underline{\mathrm{H}}\right), 7.31-7.40$ (m, 4H, $\left.\mathrm{Ar} \underline{\mathrm{H}}\right), 7.91$ (bs, $2 \mathrm{H}$, $\mathrm{Ar} \underline{\mathrm{H}}) ;{ }^{13} \mathrm{C}$ NMR $\left(75 \mathrm{MHz}, \mathrm{CDCl}_{3}\right): \delta 120.72,121.56,127.20,127.87,128.43,133.72,134.83$, 148.95, 153.09; m/z $230\left(\mathrm{M}^{+}\right)$; Anal. Calcd for $\mathrm{C}_{13} \mathrm{H}_{11} \mathrm{ClN}_{2}$ requires: $\mathrm{C}, 67.68 ; \mathrm{H}, 4.81 ; \mathrm{N}, 12.14$. Found: C, 67.62; H, 4.75; N, 12.09 .

\section{Acknowledgements}

We are grateful to Mr. B. S. Kalshetti for IR spectra, Mrs. J. P. Choudhary for NMR spectra, and Mr. D. S. Shishupal for GCMS. N. L. C. is thankful to BARC for the award of a fellowship

\section{References}

1. (a) Dunn, P. J. In Comprehensive Organic Functional Group Transformations II; Katritzky, A. R., Taylor, R. J., Eds.; Elsevier: Oxford, 2005; Vol. 5, pp 655-698. (b) Yokoyama, M.; Menjo, Y.; Wei, H.; Togo, H. Bull. Chem. Soc. Jpn. 1995, 68, 2735. (c) Lerestif, J. M.; Bazureau, J. P.; Hamelin, J. Tetrahedron Lett. 1993, 34, 4639. (d) Doise, M.; Blondeau, D.; 
Sliwa, H. Synth. Commun. 1992, 22, 2891. (e) Neunhoeffer, H.; Karafiat, U.; Köhler, G.; Sowa, B. Liebigs Ann. Chem. 1992, 115. (f) Alanine, A. I. D.; Fishwick, C. W. G. Tetrahedron Lett. 1989, 30, 4443.

2. (a) Özden, S.; Atabey, D.; Yıldız, S.; Göker, H. Bioorg. Med. Chem. 2005, 13, 1587. (b) Huang, T. L.; Tao, B.; Quarshie, Y.; Queener, S. F.; Donkor, I. O. Bioorg. Med. Chem. Lett. 2001, 11, 2679. (c) Stephens, C. E.; Tanious, F.; Kim, S.; Wilson, W. D.; Schell, W. A.; Perfect, J. R.; Franzblau, S. G.; Boykin, D. W. J. Med. Chem. 2001, 44, 1741.

3. (a) Wang, J.; Xu, F.; Cai, T.; Shen, Q. Org. Lett. 2008, 10, 445. (b) Thomas, K. K.; Reshmy, R.; Ushadevi, K. S. J. Indian Chem. Soc. 2007, 84, 1016. (c) Anbazhagan, M.; Boykin, D. W.; Stephens C. E. Tetrahedron Lett. 2002, 43, 9089. (d) Zang, J. M.; Zang, Y. M. Chinese Chem. Lett. 2002, 13, 97. (e) Fersberg, J. H.; Spaziano, V. T.; Balasubramanian, T. M. J. Org. Chem. 1987, 52, 1017. (f) Xu, F.; Sun, J.; Shen, Q. Tetrahedron Lett. 2002, 43, 1867. (g) Rousselet, G.; Capdevielle, P.; Maumy, M. Tetrahedron Lett. 1993, 34, 6395. (h) Fang, L.; Wu, H. Ziran Kexueban 2003, 26, 262. (CAN 140:423440) (i) Gandolfi, R.; Gamba, A.; Gruenanger, P. Heterocycles 1995, 40, 619. (j) Rodríguez, H.; Pavez, H.; Márquez, A.; Navarrete, P. Tetrahedron 1983, 39, 23. (k) Dondoni, A.; Barbaro, G. J. Chem. Soc., Chem. Comm. 1975, 18, 761. (1) Li, Z. F.; Lu, P.; Zang, Y. M. Chinese Chem. Lett. 2000, 11, 495.

4. Kusurkar, R. S.; Wadia, M. S.; Bhosale, D. K.; Tavale, S. S.; Puranik, V. G, J. Chem. Research (s) 1996, 478.

5. Vyas, S. M. Ph. D. thesis, University of Pune, 2002.

6. (a) Alcaide, B.; Mardomingo, C. L.; Plumet, J.; Cativiela, C.; Mayoral, J. A. Can. J. Chem. 1987, 65, 2050. (b) Jäger, V.; Colinas, P.A. In Synthetic Applications of 1,3-Dipolar Cycloaddition Chemistry toward Heterocycles and Natural Products; Padwa, A.; Pearson, W.H. Eds., Wiley: New York, 2002; Ch. 6. (c) Feuer, H., Nitrile Oxides, Nitrones and Nitronates in Organic Synthesis, 2nd Edn.; Wiley: New Jersey, 2008.

7. Bolton, R. E.; Coote, S. J.; Finch, H.; Lowdon, A.; Pegg, N.; Vinader M. V. Tetrahedron Lett. 1995, 36, 4471.

8. Yang, H. J.; Sun, W. H.; Li, Z. L.; Ma, Z. Chinese Chem. Lett. 2002, 13, 3.

9. Kazuho, H.; Eisuke, K.; Shonosuke, Z. Chem. Pharm. Bull. 1980, 28, 3296.

10. Mangat, R.; Baljit, K. J. Indian Chem. Soc. 1982, 59, 1197.

11. Benito, A.; Carmen, L. M.; Joaquin, P.; Carlos, C.; Mayoral, J. A. Can. J. Chem. 1987, 65, 2050.

12. Alessandro, D.; Gaetano, B. Chem. Comm. 1975, 18, 761.

13. Wiglend, T.; Gust, R. J. Med. Chem. 2007, 50, 1475.

14. Charlton, P. T.; Maliphant, G. M.; Oxley, P.; Peak, D. A. J. Chem. Soc. 1951, 485. 